Thorax (1964), 19, 332.

\title{
Spontaneous rupture of the pharynx
}

\author{
J A M ES S. D A V I D SON \\ From Bradford Royal Infirmary
}

Spontaneous rupture of the oesophagus is well known to all thoracic surgeons. Spontaneous rupture of the stomach, though rare, is recognized, especially in the new-born. It is perhaps surprising that, as far as we are aware, spontaneous rupture of the pharynx has not previously been described. The history and clinical findings in the following case report are consistent with this diagnosis.

\section{CASE HISTORY}

The patient was an obese housewife aged 50 years. The day her pharynx ruptured, her lunch consisted of chips and gravy followed by a steamed fruit pudding which she had made herself. To use her own words, she 'swallowed a mouthful of pudding unexpectedly'. The pudding seemed to stick in her throat and she was immediately seized with intense pain on the left side of the neck. She was at once aware also of a swelling about the size of an egg on the left side of the neck. She thrust her fingers down her throat to induce vomiting and brought up some blood. The pain became even worse and the swelling increased.

She was rushed to hospital on the pillion of a motor cycle. This was her first experience of this mode of transport.

At the hospital, which she reached one hour after the onset of pain and swelling, she was found to be moderately shocked. She complained of agonizing pain all round her neck, between the shoulder blades, and over the right shoulder blade. There was extensive surgical emphysema of the neck. The pharynx was inspected but no cause was found to account for her condition.

She was transferred to the Thoracic Surgical Unit of the Bradford Royal Infirmary about four hours after the beginning of the episode. The pain was so severe that she was unco-operative. She said she just wanted to die. Her blood pressure was $130 / 70 \mathrm{~mm}$. Hg. (The blood pressure recorded after recovery was $180 / 110 \mathrm{~mm}$. Hg.) There was gross swelling due to surgical emphysema which involved both sides of the neck, the face, and the upper part of the chest anteriorly. The surgical emphysema appeared to be spreading.

The only additional history obtained at this stage was that she had suffered from chronic bronchitis and that she had had a goitre for many years.

She was given gastrografin to swallow and a radiograph of the neck was taken two minutes later. Most of the opaque medium passed down the oesophaguas but some of it remained in the neck just above the level of the cricoid cartilage. The gastrografin lodgin $\vec{g}$ in the neck was irregularly disposed and extende $\phi$ laterally on the left side.

OPERATION An endotracheal tube was passed with out difficulty, and no abnormality of the vocal cords. was seen.

An oesophagoscope was passed into the pharynx There was blood in the lower part of the pharynx What appeared to be a mucosal tear was seen oक the left side. The cricopharyngeal sphincter was not seen and no attempt was made to pass the instrument beyond the site of the lesion. When the oesophago scope was pressed a little towards the left, severaP currants entered the lumen of the pharynx apparentlo through a breach in its wall.

An incision was made along the anterior border o $\$$ the left sterno-mastoid muscle. Access to the pharyn $\overrightarrow{6}$ was impeded by the presence of an adenoma of the left lobe of the thyroid. The adenoma was oval ip shape and measured 2 in. $(50.8 \mathrm{~mm}$.) in its long axiso It lay posteriorly behind the left lobe of the thyroid and was closely applied to the upper part of the oesophagus. The thyroid adenoma was removede The left side of the pharynx and upper oesophagus were then exposed. The tissues were oedematous Beside the pharynx there was some dark-coloures fluid and some pudding. A clean longitudinal splip $1 \frac{1}{4}$ in. $(31.75 \mathrm{~mm}$.) long was found in the left postero lateral wall of the pharynx, extending from a poing immediately above the cricopharyngeal sphincter to the hyoid bone. The rent was quite straight. The edges of the torn mucosa were smooth. They pouted a little. Apart from the rent, there was no sign of any abnormality of the mucous membrane. A finger was inserted into the pharynx and also into the oesoo phagus through the cricopharyngeal sphincter. N\& foreign body was found. The mucosa felt normal. 웅

The tear in the mucous membrane was closed witbo a continuous catgut suture, and a similar suture waફ్ used to bring together the pharyngeal muscle. The only difficulty in the closure of the pharynx arose ato the upper end of the rupture which was difficult to reach until the submandibular salivary gland had beeri dissected upwards for a short distance. The extent of the mucosal tear was slightly greater than that in the muscle. The rent in the muscle coat had to be extended a little at each end in order to demonstrates accurately the limits of the mucosal rupture. 
On regaining consciousness, the patient expressed relief that the intense pain had gone.

A small tube drain to the suture line was removed after two days. The patient was given crystamycin for 10 days. She was allowed to swallow liquids on the day after operation. The post-operative course was uncomplicated.

After the operation it was possible to enquire more closely into the patient's previous history. She told a strange story. Apparently she had always had to be careful when eating. No one was allowed to speak to her during a meal. If her attention was distracted from the act of swallowing she was inclined to choke. In spite of this, she had always eaten well and taken normal food. She was over-weight. She had never suffered from anaemia. She was rather a jolly person and not given to complaining about her health.

A barium swallow was given two weeks after the operation. No abnormality was seen in the pharynx. Deglutition appeared to be normal. A small hiatal hernia was demonstrated in the Trendelenburg position but gastro-oesophageal reflux could not be produced. There were some mild tertiary contractions in the oesophagus.

\section{DISCUSSION}

It is necessary to distinguish between, on the one hand, perforation of the pharynx due to disease, foreign body, or the passage of an instrument, and, on the other, a spontaneous rupture. No disease was found in the pharynx in this patient nor was there any sign of a foreign body. The bolus consisted of soft pudding. An oesophagoscope was passed into the pharynx but no attempt was made to pass it beyond the lesion. The rupture in the pharyngeal wall was a clean longitudinal split $1 \frac{1}{4}$ in. $(31.75 \mathrm{~mm}$.) long in apparently healthy tissues. The lesion was in all respects comparable with that found in spontaneous rupture of the oesophagus.

The swelling of the neck and the onset of pain were synchronous. The sudden onset and intensity of the pain were just as dramatic as the initial symptoms of spontaneous rupture of the oesophagus, although the degree of shock was less. In the latter condition, extravasation may for a time be confined by the pleurae within the mediastinum. There may be some delay before surgical emphysema appears in the neck. In our patient, surgical emphysema developed immediately. The escaping air was not apparently confined by fascial planes.
Spontaneous rupture of a hollow viscus due to raised pressure within it often has a predilection for one particular site. Thus the oesophagus ruptures in its lower part on the left side. Whether or not there is a site of predilection for spontaneous rupture of the pharynx cannot be stated in the absence of comparable cases. However, in a case of pneumatic rupture of the oesophagus described by Cole and Burcher (1961), where a fire extinguisher accidentally discharged in the patient's face, rupture of the oesophagus occurred at the site characteristic of spontaneous rupture. Kerr, Sloan, and O'Brien (1953) describe rupture of the lower oesophagus on its left side in a child who had bitten an inflated inner tube. From the point of view of pharyngeal rupture, it is of interest, therefore, that Hood (1957) records a patient in whom rupture of the pharynx and upper oesophagus occurred as a result of blast from a fire extinguisher directed into the nose and mouth. The pharyngeal rupture occurred on its left postero-lateral wall, presumably in the same situation as the spontaneous rupture in our patient.

As to aetiology, the lesion showed all the signs of having been caused by bursting pressure from within the pharynx. It occurred on swallowing. There are three factors of possible significance. The presence of a goitre may have interfered with deglutition. The patient's strange account of her need to concentrate on the act of swallowing suggests the possibility of some kind of neuromuscular incoordination. A diagnosis of syringobulbia was not established. The radiological finding of tertiary contractions in the oesophagus on barium swallow is perhaps further evidence in favour of disordered function.

\section{SUMMARY}

A case is described of rupture of the pharynx during the act of swallowing.

Reasons are given as to why the rupture was considered to be a spontaneous one.

Aetiological factors are suggested.

\section{REFERENCES}

Cole, D. S., and Burcher, S. K. (1961). Accidental pneumatic rupture of oesophagus and stomach. Lancet, 1, 24.

Hood, R. M. (1957). Rupture of the esophagus by compressed carbon dioxide. U.S. armed Forces med. J., 8, 587.

Kerr, H. H., Sloan, H., and O'Brien, C. E. (1953). Rupture of the esophagus by compressed air. Case report. Surgery, 33, 417 . 